\title{
Optimal Control of a Two-Stage Stochastic Hybrid Manufacturing System with Poisson Arrivals and Exponential Service Times
}

\author{
Kagan Gokbayrak and Omer Selvi
}

\begin{abstract}
Extending earlier work on single-stage stochastic hybrid system models, we consider a two-stage stochastic hybrid system where the job arrivals are represented through a Poisson process, and the service times required to attain a desired physical state are exponentially distributed dependent on the controllable process rates. For the case where the costs associated with the process rates and the inventory levels are non-decreasing convex, and the process rates take values from finite sets, we show that there exist threshold policies on both inventory levels for selecting the optimal process rates at each station.
\end{abstract}

Index Terms-Stochastic, Hybrid Systems, Two-Stage, Optimal Rate Control

\section{INTRODUCTION}

The term "hybrid" is used to characterize systems that include time-driven and event-driven dynamics. The former are represented by differential (difference) equations, while the latter may be described through various frameworks used for Discrete Event Systems (DES), such as timed automata, max-plus equations, queueing networks, or Petri nets (see [1]). Broadly speaking, two categories of modeling frameworks have been proposed to study hybrid systems: Those that extend event-driven models to include time-driven dynamics; and those that extend the traditional time-driven models to include event-driven dynamics (for an overview, see [2], [3], [4], [5])

The hybrid system modeling framework used in this paper falls into the first category above and is motivated by the structure of many manufacturing systems. In these systems, discrete entities (referred to as jobs) move through a network of work-centers which process the jobs so as to change their physical characteristics according to certain specifications. Associated with each job are a physical state and a temporal state. The physical state $z_{i, j}$ evolves according to time-driven dynamics modeled through differential equations

$$
\begin{aligned}
\dot{z}_{i, j} & =f_{j}\left(z_{i, j}, u_{i, j}\right) \quad \text { for } i=1,2, \ldots \\
z_{i, j}\left(\tau_{i, j}\right) & =\zeta_{i, j}^{0} z_{i, j}\left(\tau_{i, j}+s_{i, j}\right)=\zeta_{i, j}^{d}
\end{aligned}
$$

which, depending on the particular problem being studied, describe changes in such quantities as the temperature, size, weight, chemical composition, bacteria level, or some other measure of the "quality" of the job. The temporal state of a

K. Gokbayrak and $O$. Selvi are with the Department of Industrial Engineering, Bilkent University, Ankara, Turkey kgokbayr, selvi@bilkent.edu.tr, job evolves according to event-driven dynamics, e.g., by the Lindley Equation (see in [1])

$$
\begin{array}{ll}
x_{i, 1}=\max \left(a_{i}, x_{i-1,1}\right)+s_{i, 1} & x_{0,1}=-\infty \\
x_{i, 2}=\max \left(x_{i, 1}, x_{i-1,2}\right)+s_{i, 2} & x_{0,2}=-\infty
\end{array}
$$

and includes information such as the arrival time $a_{i}$, departure times $x_{i, j}$, and service times $s_{i, j}$ of job $i$ at work-center $j$ dependent on the control input $u_{i, j}$ applied on job $i$ to bring it to a desired final state $\zeta_{i, j}^{d}$. The interaction of timedriven with event-driven dynamics leads to a natural tradeoff between temporal requirements on job completion times and physical requirements on the quality of the completed jobs. For example, while the physical state of a job can be made arbitrarily close to a desired "quality target," this usually comes at the expense of long processing times resulting in excessive inventory costs or violation of constraints on job completion deadlines. Our objective, therefore, is to formulate and solve optimal control problems associated with such tradeoffs.

In [6], [7], [8], and [9], the hybrid system framework is adopted to analyze a single-stage manufacturing process assuming a deterministic setting, i.e., a known job arrival schedule and controllable service times for all jobs. An efficient algorithm to determine the optimal service times for a class of single-stage systems is presented in [8]. In [10], however, a stochastic model of a single-stage manufacturing system is studied, where the job arrivals are represented through a Poisson process with the control variable being the exponential service's process rate. Adopting an $M / M / 1$ queueing model to describe the event driven dynamics, it is shown that when the inventory-level-dependent service process rates take values from a finite set, and the costs associated with the process rate and the inventory level are non-decreasing convex, there exists a threshold policy on the inventory level for selecting the optimal process rate. In this paper, we extend the model in [10] to a two-stage hybrid system model and show that similar threshold policies exist on inventory levels for each station.

\section{PRoblem Formulation}

Consider the two stage serial manufacturing system depicted in Figure 1. Jobs are arriving to the system according to a Poisson process with rate $\lambda$, while servers process one job 


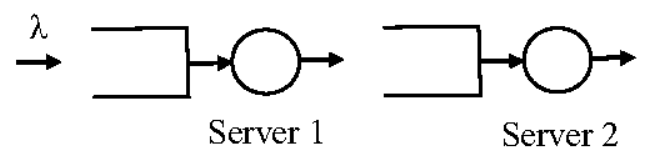

Fig. 1. Two-stage serial manufacturing system

at a time on a first-come first-served non-preemptive basis (i.e. a job in service can not be interrupted until its service completion). Service times for both servers are exponentially distributed and server $l(l=1,2)$ operates with rate $u^{l} \in U_{l}$ where $U_{l}$ 's are finite sets such that

$$
U_{l}=\left\{u_{1}^{l}, u_{2}^{l}, \ldots, u_{m_{l}}^{l}\right\}
$$

The rates $u_{i}^{l}$ are indexed so that $u_{i}^{l}<u_{i+1}^{l}$ for $i=1, \ldots, m_{l}-1$.

Defining a rate $\gamma=\lambda+u_{m_{1}}^{1}+u_{m_{2}}^{2}$, this system can be modeled as a discrete-time Markov Chain (DTMC). The state space for this DTMC can be defined as

$$
S=\left\{(i, j): i, j \in \mathbb{Z}_{0}^{+}\right\}
$$

where the state $(i, j)$ describes the system with $i$ jobs in the first server and $j$ jobs in the second server. The transition probabilities for this DTMC are

$$
\begin{gathered}
P_{(0,0),(k, l)}=\left\{\begin{array}{cc}
\frac{\lambda}{\gamma} & (k, l)=(1,0) \\
1-\frac{\lambda}{\gamma} & (k, l)=(0,0) \\
0 & \text { otherwise }
\end{array}\right. \\
P_{(i, 0),(k, l)}=\left\{\begin{array}{cc}
\frac{\lambda}{\gamma} & (k, l)=(i+1,0) \\
\frac{u^{1}(i, 0)}{\gamma} & (k, l)=(i-1,1) \\
1-\frac{\lambda+u^{1}(i, 0)}{\gamma} & (k, l)=(i, 0) \\
0 & \text { otherwise }
\end{array}\right. \\
P_{(0, j),(k, l)}=\left\{\begin{array}{cc}
\frac{\lambda}{\gamma} & (k, l)=(1, j) \\
\frac{u^{2}(0, j)}{\gamma} & (k, l)=(0, j-1) \\
1-\frac{\lambda+u^{2}(0, j)}{\gamma} & (k, l)=(0, j) \\
0 & \text { otherwise } \\
P_{(i, j),(k, l)}=\left\{\begin{array}{cc}
\frac{\lambda}{\gamma} \\
\frac{u^{1}(i, j)}{\gamma}
\end{array}\right. \\
\frac{u^{2}(i, j)}{\gamma} & (k, l)=(i+1, j)=(i-1, j+1) \\
1-\frac{\lambda+u^{1}(i, j)+u^{2}(i, j)}{\gamma} & (k, l)=(i, j-1) \\
0 & (k, l)=(i, j)
\end{array}\right. \\
\text { otherwise }
\end{gathered}
$$

Assuming that the servers start with system sizes $q_{0}^{l}$, the infinite horizon problem we consider is to determine the stationary state-dependent rate setting policy $\pi$ such that the discounted cost

$$
V_{\pi}\left(q_{0}^{1}, q_{0}^{2}\right)=E_{\pi}\left[\sum_{k=0}^{\infty} \alpha^{k} C\left(q_{k}^{1}, q_{k}^{2}, u_{k}^{1}, u_{k}^{2}\right)\right]
$$

is minimized. Note that in (6), $\alpha$ is the discount factor, $q_{k}^{l}$ denotes the system size for server $l$, and the pair

$$
\left(u_{k}^{1}, u_{k}^{2}\right)=\pi\left(q_{k}^{1}, q_{k}^{2}\right)
$$

denotes the corresponding policy-determined process rates for the servers. The one step cost $C\left(q_{k}^{1}, q_{k}^{2}, u_{k}^{1}, u_{k}^{2}\right)$ in (6) is assumed to be separable, i.e.,

$$
C\left(q_{k}^{1}, q_{k}^{2}, u_{k}^{1}, u_{k}^{2}\right)=b_{1}\left(q_{k}^{1}\right)+b_{2}\left(q_{k}^{2}\right)+c_{1}\left(u_{k}^{1}\right)+c_{2}\left(u_{k}^{2}\right)
$$

where the inventory costs $b_{l}($.$) and the service costs c_{l}($.$) are$ non-decreasing convex functions.

\section{OPtimal Rate CONTRol Policy}

Applying Dynamic Programming (DP), in e.g. [11], to solve the optimal control problem (6), the discounted cost-to-go DP equation for state $(i, j)$ becomes

$$
V_{n+1}(i, j)=\min _{u^{1}(i, j) \in U_{1}}\left\{\begin{array}{c}
b_{1}(i)+b_{2}(j) \\
u^{2}(i, j) \in U_{2} \\
+c_{1}\left(u^{1}(i, j)\right)+c_{2}\left(u^{2}(i, j)\right) \\
+\alpha \frac{\lambda}{\gamma} V_{n}(i+1, j) \\
+\alpha \frac{u^{1}(i, j)}{\gamma} V_{n}(i-1, j+1) \\
+\alpha \frac{u^{2}(i, j)}{\gamma} V_{n}(i, j-1) \\
+\alpha V_{n}(i, j) \\
-\alpha \frac{\lambda+u^{1}(i, j)+u^{2}(i, j)}{\gamma} V_{n}(i, j)
\end{array}\right\}
$$

Note that for all $i, j, n \in \mathbb{Z}_{0}^{+}$, we have assumed

$$
V_{n}(-1, j+1)=V_{n}(0, j)
$$

and

$$
V_{n}(i,-1)=V_{n}(i, 0)
$$

Let us define

$$
\begin{aligned}
& \Delta V_{n}^{(1)}(i, j)=V_{n}(i, j)-V_{n}(i-1, j+1) \\
& \Delta V_{n}^{(2)}(i, j)=V_{n}(i, j)-V_{n}(i, j-1)
\end{aligned}
$$

By (8) and (9), for all $i, j, n \in \mathbb{Z}_{0}^{+}$

$$
\Delta V_{n}^{(1)}(0, j)=\Delta V_{n}^{(2)}(i, 0)=0
$$

The following theorem establishes the optimal control policy:

Theorem 1: The optimal controls for the $(n+1)$ th step are

$$
\begin{aligned}
u_{n+1}^{1}(i, j)= & \arg \min _{u^{1} \in U_{1}}\left\{c_{1}\left(u^{1}\right)-\alpha \frac{u^{1}}{\gamma} \Delta V_{n}^{(1)}(i, j)\right\} \\
u_{n+1}^{2}(i, j)= & \arg \min _{u^{2} \in U_{2}}\left\{c_{2}\left(u^{2}\right)-\alpha \frac{u^{2}}{\gamma} \Delta V_{n}^{(2)}(i, j)\right\} \\
\text { Proof: The cost-to-go equation in }(7) \text { can be written as } & \\
V_{n+1}(i, j)= & b_{1}(i)+b_{2}(j)+\alpha V_{n}(i, j) \\
& +\alpha \frac{\lambda}{\gamma}\left[V_{n}(i+1, j)-V_{n}(i, j)\right] \\
& +\min _{u^{1}(i, j) \in U_{1}}\left\{-\alpha \frac{u^{1}(i, j)}{\gamma} \Delta V_{n}^{(1)}(i, j)\right\} \\
& +\min _{u^{2}(i, j) \in U_{2}}\left\{-\alpha \frac{c^{2}(i, j)}{\gamma} \Delta V_{n}^{(2)}(i, j)\right\}
\end{aligned}
$$


Hence, the result follows.

Corollary 1: The optimal rates for empty servers are the lowest cost rates for those servers, i.e.,

$$
\begin{aligned}
& u^{1}(0, j)=\arg \min _{u^{1} \in U_{1}}\left\{c_{1}\left(u^{1}\right)\right\}=u_{1}^{1} \\
& u^{2}(i, 0)=\arg \min _{u^{2} \in U_{2}}\left\{c_{2}\left(u^{2}\right)\right\}=u_{1}^{2}
\end{aligned}
$$

Proof: Since $\Delta V_{n}^{(1)}(0, j)$ and $\Delta V_{n}^{(2)}(i, 0)$ are zero for all $i, j \in \mathbb{Z}_{0}^{+}$, the result follows from Theorem 1 .

Since it is computationally impossible to solve for $\Delta V_{n}^{(1)}(i, j)$ and $\Delta V_{n}^{(2)}(i, j)$ over all $i, j$ and $n$, determining the optimal process rates for all states $(i, j)$ analytically is not feasible. Instead, we will exploit monotonicity properties of $\Delta V_{n}^{(1)}(i, j)$ and $\Delta V_{n}^{(2)}(i, j)$ to establish inventory level thresholds for the optimal process rates.

Using notation $c_{k}^{l}=c_{l}\left(u_{k}^{l}\right)$, we can define the thresholds $\beta_{k}^{l}$ as

$$
\beta_{k}^{l}=\left\{\begin{array}{cc}
0 & k=0 \\
\frac{\gamma}{\alpha}\left(\frac{c_{k+1}^{l}-c_{k}^{l}}{u_{k+1}^{l}-u_{k}^{l}}\right) & 0<k<m \\
\infty & k=m_{l}
\end{array}\right.
$$

Given that $c_{l}($.$) is a non-decreasing convex function, \beta_{k}^{l}$ is also non-decreasing in $k$ for $l=1,2$, which allows us to establish the following optimality condition:

Lemma 1: The optimal process rate $u^{l}(i, j)=u_{k}^{l}$ if and only if $\beta_{k-1}^{l} \leq \Delta V_{n}^{(l)}(i, j) \leq \beta_{k}^{l}$

Proof: $(\Leftarrow)$ Let us assume that

$$
\beta_{k-1}^{l} \leq \Delta V_{n}^{(l)}(i, j) \leq \beta_{k}^{l}
$$

and

$$
u^{l}(i, j)=u_{t}^{l} \neq u_{k}^{l}
$$

Then,

$$
c_{t}^{l}-\alpha \frac{u_{t}^{l}}{\gamma} \Delta V_{n}^{(l)}(i, j)<c_{k}^{l}-\alpha \frac{u_{k}^{l}}{\gamma} \Delta V_{n}^{(l)}(i, j)
$$

If $t>k$ then

$$
\beta_{k}^{l} \leq \frac{\gamma}{\alpha} \frac{c_{t}^{l}-c_{k}^{l}}{u_{t}^{l}-u_{k}^{l}}<\Delta V_{n}^{(l)}(i, j)
$$

If $t<k$ then

$$
\beta_{k-1}^{l} \geq \frac{\gamma}{\alpha} \frac{c_{k}^{l}-c_{t}^{l}}{u_{k}^{l}-u_{t}^{l}}>\Delta V_{n}^{(i)}(i, j)
$$

Both cases contradict our assumption, so the optimal process rate $u^{l}(i, j)=u_{k}^{l}$.

$\Leftrightarrow)$ Conversely, let us assume that $u^{l}(i, j)=u_{k}^{l}$ and consider the following cases: If

$$
\Delta V_{n}^{(l)}(i, j)>\beta_{k}^{l}=\frac{\gamma}{\alpha}\left(\frac{c_{k+1}^{l}-c_{k}^{l}}{u_{k+1}^{l}-u_{k}^{l}}\right)
$$

then

$$
c_{k}^{l}-\alpha \frac{u_{k}^{l}}{\gamma} \Delta V_{n}^{(l)}(i, j)>c_{k+1}^{l}-\alpha \frac{u_{k+1}^{l}}{\gamma} \Delta V_{n}^{(l)}(i, j)
$$

which contradicts the $u^{l}(i, j)=u_{k}^{l}$ assumption. Similarly, if

$$
\Delta V_{n}^{(l)}(i, j)<\beta_{k-1}^{l}=\frac{\gamma}{\alpha}\left(\frac{c_{k}^{l}-c_{k-1}^{l}}{u_{k}^{l}-u_{k-1}^{l}}\right)
$$

then

$$
c_{k-1}^{l}-\alpha \frac{u_{k-1}^{l}}{\gamma} \Delta V_{n}^{(l)}(i, j)<c_{k}^{l}-\alpha \frac{u_{k}^{l}}{\gamma} \Delta V_{n}^{(l)}(i, j)
$$

which contradicts the $u^{l}(i, j)=u_{k}^{l}$ assumption.

An immediate corollary of Lemma 1 is the following: If $\Delta V_{n}^{(l)}\left(i_{1}, j_{1}\right) \leq \Delta V_{n}^{(l)}\left(i_{2}, j_{2}\right)$ then the optimal process rates satisfy $u^{l}\left(i_{1}, j_{1}\right) \leq u^{l}\left(i_{2}, j_{2}\right)$. Depending on the $\left[\beta_{k-1}^{l}, \beta_{k}^{l}\right]$ interval that the $\Delta V_{n}^{(l)}(i, j)$ values fall in, an optimal process rate $u_{k}^{l}$ is selected.

The following theorem establishes monotonicity properties of the $\Delta V_{n}^{(l)}(i, j)$ and implies the existence of a threshold policy.

Theorem 2: Given $b($.$) and c($.$) as non-decreasing convex$ functions, for all $i, j, n \in \mathbb{Z}_{0}^{+}$.

i) $\Delta V_{n}^{(1)}(i, j)$ is non-decreasing in $i$ and non-increasing in $j$, i.e.,

$$
\begin{aligned}
& \Delta V_{n}^{(1)}(i, j) \leq \Delta V_{n}^{(1)}(i+1, j) \\
& \Delta V_{n}^{(1)}(i, j) \geq \Delta V_{n}^{(1)}(i, j+1)
\end{aligned}
$$

ii) $\Delta V_{n}^{(2)}(i, j)$ is non-decreasing in both $i$ and $j$, i.e.,

$$
\begin{aligned}
& \Delta V_{n}^{(2)}(i, j) \leq \Delta V_{n}^{(2)}(i+1, j) \\
& \Delta V_{n}^{(2)}(i, j) \leq \Delta V_{n}^{(2)}(i, j+1)
\end{aligned}
$$

Proof: (By Induction)

Let us define

$$
\begin{aligned}
& \delta V_{k, i, j}^{l}(1)=\Delta V_{k}^{(l)}(i+1, j)-\Delta V_{k}^{(l)}(i, j) \\
& \delta V_{k, i, j}^{l}(2)=\Delta V_{k}^{(l)}(i, j+1)-\Delta V_{k}^{(l)}(i, j)
\end{aligned}
$$

We need to show that for all $k$

$$
\begin{aligned}
& \delta V_{k, i, j}^{1}(1) \geq 0, \delta V \quad \stackrel{1}{k, i, j}(2) \leq 0 \\
& \delta V_{k, i, j}^{2}(1) \geq 0, \delta V \quad \underset{k, i, j}{2}(2) \geq 0
\end{aligned}
$$

For $k=0$, since $V_{0}(i, j)=0$ for all $i, j$,

$$
\begin{array}{lll}
\delta V_{0, i, j}^{1}(1) & =0, \delta V & 1 \\
0, i, j & (2)=0 \\
\delta V_{0, i, j}^{2}(1) & =0, \delta V & 2, i, j \\
0,2 & =0
\end{array}
$$

Next, assume that for $k=n$, for all $i, j$

$$
\begin{aligned}
& \delta V_{n, i, j}^{1}(1) \geq 0, \delta V \quad \underset{n, i, j}{1}(2) \leq 0 \\
& \delta V_{n, i, j}^{2}(1) \geq 0, \delta V \quad \underset{n, i, j}{2}(2) \geq 0
\end{aligned}
$$

We need to show that for $k=n+1$ and for all $i, j$

$$
\begin{aligned}
& \delta V_{n+1, i, j}^{1}(1) \geq 0, \delta V \quad \begin{array}{l}
1 \\
n+1, i, j
\end{array}(2) \leq 0 \\
& \delta V_{n+1, i, j}^{2}(1) \geq 0, \delta V \quad \underset{n+1, i, j}{2}(2) \geq 0
\end{aligned}
$$

inequalities are satisfied. 


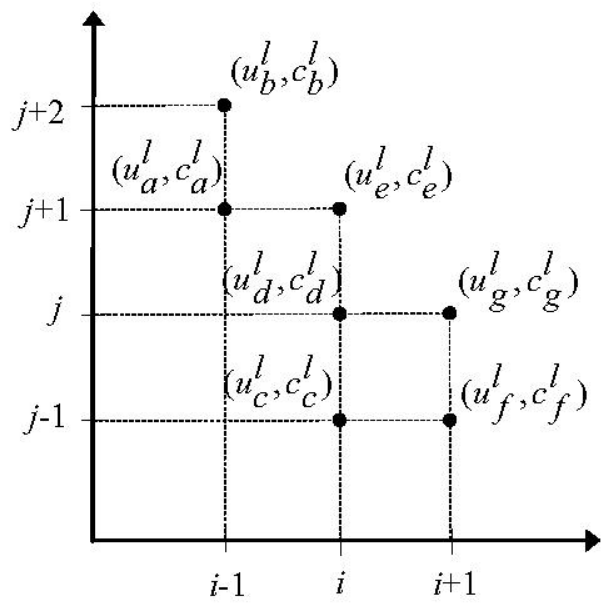

Fig. 2. Optimal process rates and their costs for step $(n+1)$

Let us assume that optimal process rates from Theorem 1 and their costs are calculated (given in Figure 2.) Then, $\Delta V_{n+1}^{(1)}(i, j)$ and $\Delta V_{n+1}^{(2)}(i, j)$ can be given as

$$
\begin{aligned}
\Delta V_{n+1}^{(1)}(i, j)= & b_{1}(i)-b_{1}(i-1)+b_{2}(j)-b_{2}(j+1) \\
& +\alpha \Delta V_{n}^{(1)}(i, j)+\alpha \frac{\lambda}{\gamma} \delta V_{n, i, j}^{1}(1) \\
& +c_{d}^{1}-\alpha \frac{u_{d}^{1}}{\gamma} \Delta V_{n}^{(1)}(i, j) \\
& -c_{a}^{1}+\alpha \frac{u_{a}^{1}}{\gamma} \Delta V_{n}^{(1)}(i-1, j+1) \\
& +c_{d}^{2}-\alpha \frac{u_{d}^{2}}{\gamma} \Delta V_{n}^{(2)}(i, j) \\
& -c_{a}^{2}+\alpha \frac{u_{a}^{2}}{\gamma} \Delta V_{n}^{(2)}(i-1, j+1) \\
\Delta V_{n+1}^{(2)}(i, j)= & b_{2}(j)-b_{2}(j-1)+\alpha \Delta V_{n}^{(2)}(i, j) \\
& +\alpha \frac{\lambda}{\gamma} \delta V_{n, i, j}^{2}(1)+c_{d}^{1}-\alpha \frac{u_{d}^{1}}{\gamma} \Delta V_{n}^{(1)}(i, j) \\
& -c_{c}^{1}+\alpha \frac{u_{c}^{1}}{\gamma} \Delta V_{n}^{(1)}(i, j-1) \\
& +c_{d}^{2}-\alpha \frac{u_{d}^{2}}{\gamma} \Delta V_{n}^{(2)}(i, j) \\
& -c_{c}^{2}+\alpha \frac{u_{c}^{2}}{\gamma} \Delta V_{n}^{(2)}(i, j-1)
\end{aligned}
$$

Note that by the induction hypothesis for all $i, j$

$$
\begin{aligned}
& \Delta V_{n}^{(2)}(i, j+1)-\Delta V_{n}^{(2)}(i+1, j) \\
= & {\left[V_{n}(i, j+1)-V_{n}(i, j)\right] } \\
& -\left[V_{n}(i+1, j)-V_{n}(i+1, j-1)\right] \\
= & {\left[V_{n}(i+1, j-1)-V_{n}(i, j)\right] } \\
& -\left[V_{n}(i+1, j)-V_{n}(i, j+1)\right] \\
= & -\delta V_{n, i+1, j-1}^{1}(2) \geq 0
\end{aligned}
$$

hence by Lemma $1 u_{n+1}^{2}(i+1, j) \leq u_{n+1}^{2}(i, j+1)$ holds for all $i, j$. This enables us to claim the following inequalities:

$$
\begin{aligned}
& u_{b}^{1} \leq u_{a}^{1} \leq u_{e}^{1} \leq u_{d}^{1} \leq u_{g}^{1} \leq u_{f}^{1} \\
& u_{d}^{1} \leq u_{c}^{1} \leq u_{f}^{1} \\
& u_{c}^{2} \leq u_{f}^{2} \leq u_{d}^{2} \leq u_{g}^{2} \leq u_{e}^{2} \leq u_{b}^{2} \\
& u_{d}^{2} \leq u_{a}^{2} \leq u_{e}^{2}
\end{aligned}
$$

Step 1: (Proof of $\delta V_{n+1, i, j}^{1}(1) \geq 0$ )

We have

$$
\begin{aligned}
\delta V_{n+1, i, j}^{1}(1)= & {\left[b_{1}(i+1)-b_{1}(i)\right]-\left[b_{1}(i)-b_{1}(i-1)\right] } \\
& +\alpha \frac{\lambda}{\gamma} \delta V_{n, i+1, j}^{1}(1)+T_{1}^{(1)}+T_{2}^{(1)}
\end{aligned}
$$

where

$$
\begin{aligned}
T_{1}^{(1)}= & \alpha \frac{u_{m_{1}}^{1}-u_{g}^{1}}{\gamma} \delta V_{n, i, j}^{1}(1)+\alpha \frac{u_{a}^{1}}{\gamma} \delta V_{n, i-1, j+1}^{1}(1) \\
& +\left[\left(c_{g}^{1}-c_{d}^{1}\right)-\alpha \frac{u_{g}^{1}-u_{d}^{1}}{\gamma} \Delta V_{n}^{(1)}(i, j)\right] \\
& -\left[\left(c_{e}^{1}-c_{a}^{1}\right)-\alpha \frac{u_{e}^{1}-u_{a}^{1}}{\gamma} \Delta V_{n}^{(1)}(i, j+1)\right] \\
T_{2}^{(1)}= & \alpha \frac{u_{m_{2}}^{2}-u_{g}^{2}}{\gamma} \delta V_{n, i, j}^{1}(1)+\alpha \frac{u_{g}^{2}}{\gamma} \delta V_{n, i, j-1}^{1}(1) \\
& +\alpha \frac{u_{a}^{2}-u_{g}^{2}}{\gamma} \delta V_{n, i-1, j+1}^{2}(1) \\
& +\left[\left(c_{g}^{2}-c_{d}^{2}\right)-\alpha \frac{u_{g}^{2}-u_{d}^{2}}{\gamma} \Delta V_{n}^{(2)}(i, j)\right] \\
& -\left[\left(c_{e}^{2}-c_{a}^{2}\right)-\alpha \frac{u_{e}^{2}-u_{a}^{2}}{\gamma} \Delta V_{n}^{(2)}(i, j+1)\right]
\end{aligned}
$$

Note that we can manipulate (12) to derive an equivalent definition for $T_{2}^{(1)}$ :

$$
\begin{aligned}
T_{2}^{(1)}= & \alpha \frac{u_{m_{2}}^{2}-u_{a}^{2}}{\gamma} \delta V_{n, i, j}^{1}(1)+\frac{\alpha}{\gamma} u_{a}^{2} \delta V_{n, i, j-1}^{1}(1) \\
& -\alpha \frac{u_{g}^{2}-u_{a}^{2}}{\gamma} \delta V_{n, i+1, j-1}^{1}(2) \\
& +\left[\left(c_{a}^{2}-c_{d}^{2}\right)-\frac{\alpha}{\gamma}\left(u_{a}^{2}-u_{d}^{2}\right) \Delta V_{n}^{(2)}(i, j)\right] \\
& -\left[\left(c_{e}^{2}-c_{g}^{2}\right)-\frac{\alpha}{\gamma}\left(u_{e}^{2}-u_{g}^{2}\right) \Delta V_{n}^{(2)}(i, j+1)\right]
\end{aligned}
$$

Observe that

1. Since $b_{1}($.$) is non-decreasing convex in i$,

$$
\left[b_{1}(i+1)-b_{1}(i)\right]-\left[b_{1}(i)-b_{1}(i-1)\right] \geq 0
$$

2. By the induction hypothesis,

$$
\alpha \frac{\lambda}{\gamma} \delta V_{n, i+1, j}^{1}(1) \geq 0
$$

3. By the induction hypothesis, and since $u_{m_{1}}^{1} \geq u_{g}^{1}$,

$$
\alpha \frac{u_{m_{1}}^{1}-u_{g}^{1}}{\gamma} \delta V_{n, i, j}^{1}(1)+\alpha \frac{u_{a}^{1}}{\gamma} \delta V_{n, i-1, j+1}^{1}(1) \geq 0
$$


4. a. If $u_{g}^{1}=u_{d}^{1}$, then

$$
\left(c_{g}^{1}-c_{d}^{1}\right)-\alpha \frac{u_{g}^{1}-u_{d}^{1}}{\gamma} \Delta V_{n}^{(1)}(i, j)=0
$$

4. b. If $u_{g}^{1}>u{ }_{d}^{1}$, then

$$
\Delta V_{n}^{(1)}(i, j) \leq \beta_{d}^{1} \leq \frac{\gamma}{\alpha} \frac{\left(c_{g}^{1}-c_{d}^{1}\right)}{\left(u_{g}^{1}-u_{d}^{1}\right)}
$$

Hence,

$$
\left(c_{g}^{1}-c_{d}^{1}\right)-\alpha \frac{u_{g}^{1}-u_{d}^{1}}{\gamma} \Delta V_{n}^{(1)}(i, j) \geq 0
$$

5. a. If $u_{e}^{1}=u_{a}^{1}$, then

$$
\left(c_{e}^{1}-c_{a}^{1}\right)-\alpha \frac{u_{e}^{1}-u_{a}^{1}}{\gamma} \Delta V_{n}^{(1)}(i, j+1)=0
$$

5. b. If $u_{e}^{1}>u_{a}^{1}$, then

$$
\Delta V_{n}^{(1)}(i, j+1) \geq \beta_{e-1}^{1} \geq \frac{\gamma}{\alpha} \frac{\left(c_{e}^{1}-c_{a}^{1}\right)}{\left(u_{e}^{1}-u_{a}^{1}\right)}
$$

Hence,

$$
\left(c_{e}^{1}-c_{a}^{1}\right)-\alpha \frac{u_{e}^{1}-u_{a}^{1}}{\gamma} \Delta V_{n}^{(1)}(i, j+1) \leq 0
$$

From 3, 4, and 5, we establish that $T_{1}^{(1)} \geq 0$. In order to show that $\delta V_{n+1, i, j}^{1}(1) \geq 0$, we will also show $T_{2}^{(1)} \geq 0$. For this purpose we will consider the $u_{g}^{2} \leq u_{a}^{2}$ case with (12) and the $u_{g}^{2}>u_{a}^{2}$ case with (13).

Case 1: $u_{a}^{2} \leq u_{a}^{2}$

6. By the induction hypothesis, and that $u_{g}^{2} \leq u_{a}^{2} \leq u_{m_{2}}^{2}$,

$$
\begin{aligned}
\alpha \frac{u_{m_{2}}^{2}-u_{g}^{2}}{\gamma} \delta V_{n, i, j}^{1}(1)+\alpha \frac{u_{g}^{2}}{\gamma} \delta V_{n, i, j-1}^{1}(1) & \geq 0 \\
\alpha \frac{u_{a}^{2}-u_{g}^{2}}{\gamma} \delta V_{n, i-1, j+1}^{2}(1) & \geq 0
\end{aligned}
$$

7. a. If $u_{g}^{2}=u_{d}^{2}$, then

$$
\left(c_{g}^{2}-c_{d}^{2}\right)-\alpha \frac{u_{g}^{2}-u_{d}^{2}}{\gamma} \Delta V_{n}^{(2)}(i, j)=0
$$

7. b. If $u_{g}^{2}>u_{d}^{2}$, then

$$
\Delta V_{n}^{(2)}(i, j) \leq \beta_{d}^{2} \leq \frac{\gamma}{\alpha} \frac{\left(c_{g}^{2}-c_{d}^{2}\right)}{\left(u_{g}^{2}-u_{d}^{2}\right)}
$$

Hence,

$$
\left(c_{g}^{2}-c_{d}^{2}\right)-\alpha \frac{u_{g}^{2}-u_{d}^{2}}{\gamma} \Delta V_{n}^{(2)}(i, j) \geq 0
$$

8. a. If $u_{e}^{2}=u_{a}^{2}$, then

$$
\left(c_{e}^{2}-c_{a}^{2}\right)-\alpha \frac{u_{e}^{2}-u_{a}^{2}}{\gamma} \Delta V_{n}^{(2)}(i, j+1)=0
$$

8. b. If $u_{e}^{2}>u_{a}^{2}$, then

$$
\Delta V_{n}^{(2)}(i, j+1) \geq \beta_{e-1}^{2} \geq \frac{\gamma}{\alpha} \frac{\left(c_{e}^{2}-c_{a}^{2}\right)}{\left(u_{e}^{2}-u_{a}^{2}\right)}
$$

Hence,

$$
\left(c_{e}^{2}-c_{a}^{2}\right)-\alpha \frac{u_{e}^{2}-u_{a}^{2}}{\gamma} \Delta V_{n}^{(2)}(i, j+1) \leq 0
$$

Case 2: $u_{g}^{2}>u_{a}^{2}$

9. By the induction hypothesis, by $u_{a}^{2}<u_{g}^{2} \leq u_{m_{2}}^{2}$, and by the argument in (11)

$$
\begin{aligned}
\alpha \frac{u_{m_{2}}^{2}-u_{a}^{2}}{\gamma} \delta V_{n, i, j}^{1}(1)+\frac{\alpha}{\gamma} u_{a}^{2} \delta V_{n, i, j-1}^{1}(1) & \geq 0 \\
-\alpha \frac{u_{g}^{2}-u_{a}^{2}}{\gamma} \delta V_{n, i+1, j-1}^{1}(2) & \geq 0
\end{aligned}
$$

10. a. If $u_{a}^{2}=u_{d}^{2}$, then

$$
\left(c_{a}^{2}-c_{d}^{2}\right)-\frac{\alpha}{\gamma}\left(u_{a}^{2}-u_{d}^{2}\right) \Delta V_{n}^{(2)}(i, j)=0
$$

10. b. If $u_{a}^{2}>u_{d}^{2}$

$$
\Delta V_{n}^{(2)}(i, j) \leq \beta_{d}^{2} \leq \frac{\gamma}{\alpha} \frac{\left(c_{a}^{2}-c_{d}^{2}\right)}{\left(u_{a}^{2}-u_{d}^{2}\right)}
$$

Hence,

$$
\left(c_{a}^{2}-c_{d}^{2}\right)-\frac{\alpha}{\gamma}\left(u_{a}^{2}-u_{d}^{2}\right) \Delta V_{n}^{(2)}(i, j) \geq 0
$$

11. a. If $u_{e}^{2}=u_{g}^{2}$, then

$$
\left(c_{e}^{2}-c_{g}^{2}\right)-\frac{\alpha}{\gamma}\left(u_{e}^{2}-u_{g}^{2}\right) \Delta V_{n}^{(2)}(i, j+1)=0
$$

11. b. If $u_{e}^{2}>u_{g}^{2}$, then

$$
\Delta V_{n}^{(2)}(i, j+1) \geq \beta_{e-1}^{2} \geq \frac{\gamma}{\alpha} \frac{\left(c_{e}^{2}-c_{g}^{2}\right)}{\left(u_{e}^{2}-u_{g}^{2}\right)}
$$

Hence,

$$
\left(c_{e}^{2}-c_{g}^{2}\right)-\alpha \frac{u_{e}^{2}-u_{g}^{2}}{\gamma} \Delta V_{n}^{(2)}(i, j+1) \leq 0
$$

For both cases we showed that $T_{2}^{(1)} \geq 0$ completing this step, proving that $\delta V_{n+1, i, j}^{1}(1) \geq 0$.

Other steps of the proof are similar and omitted for space considerations. The complete proof is given in [12].

It follows from Lemma 1 and Theorem 2 that an inventory level threshold policy is optimal for determining the process rates. Such thresholds are depicted in the following numerical example.

Note that the three possible events may affect the process rates as follows: an arrival to the system has the potential of increasing the process rates for both servers, a departure from the first server has the potential of decreasing the process rate for the first server and increasing the process rate for the second server, and a departure from the second server has the potential of increasing the process rate for the first server and decreasing the process rate for the second server. 


\section{NUMERICAL EXAMPLE}

Let the arrival rate to the two-stage serial manufacturing system be $\lambda=17$ while the service rate sets and respective costs of operation for both stages are

$$
\begin{array}{rll}
U_{1} & =\{30,50,70\} \\
c_{1}(30) & =4, c \quad{ }_{1}(50)=7, c \quad & 1(70)=12 \\
U_{2} & =\{40,60,90\} \\
c_{2}(40) & =2, c \quad{ }_{2}(60)=6, c \quad{ }_{2}(90)=15
\end{array}
$$

The costs of holding $(i, j)$ inventory is

$$
b_{1}(i)=3 i, b \quad{ }_{2}(j)=5 j
$$

The optimal process rates obtained by the Genetic Algorithm (see in [13]) are shown in Figure 3 for the first server and in Figure 4 for the second server:

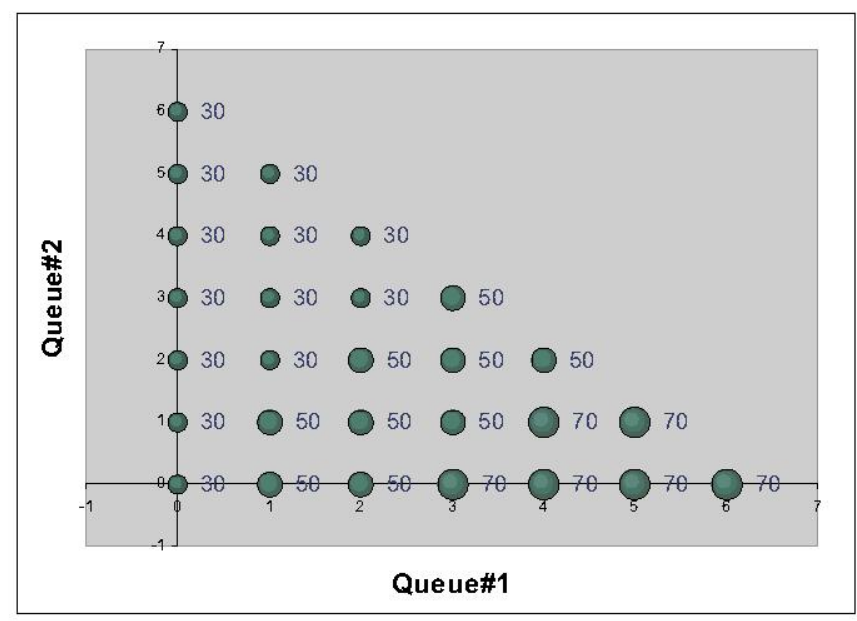

Fig. 3. Optimal process rates for server 1

\section{CONCLUSION}

In this study, we modeled a stochastic two-stage manufacturing system with two $\mathrm{M} / \mathrm{M} / 1$ queueing systems in series. The controllable process rates for both stations took values from finite sets. For the case where the single step costs associated with the process rates and the inventory levels are non-decreasing convex, we show that there exist threshold policies on both inventory levels for selecting the optimal process rates.

Extending these results for the $\mathrm{N}$-machine series production line is the subject of ongoing research.

\section{REFERENCES}

[1] C. G. Cassandras and S. Lafortune, Introduction to Discrete Event Systems. Kluwer Academic Publishers, 1999.

[2] A. Alur, T. A. Henzinger, and E. D. Sontag, eds., Hybrid Systems. Springer-Verlag, 1996.

[3] P. Antsaklis, W. Kohn, M. Lemmon, A. Nerode, and S. Sastry, eds., Hybrid Systems. Springer-Verlag, 1998.

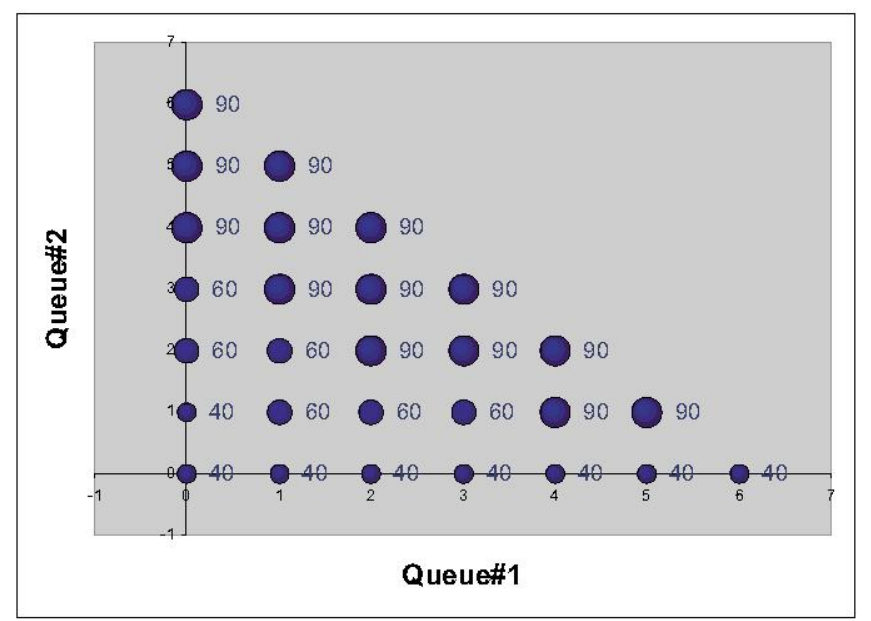

Fig. 4. Optimal process rates for server 2

[4] M. S. Branicky, V. S. Borkar, and S. K. Mitter, "A unified framework for hybrid control: Model and optimal control theory," IEEE Tr. on Automatic Control, vol. 43, no. 1, pp. 31-45, 1998.

[5] R. L. Grossman, A. Nerode, A. P. Ravn, and H. Rischel, eds., Hybrid Systems - Vol. 736 of Lecture Notes in Computer Science. SpringerVerlag, 1993.

[6] Y. Wardi, C. G. Cassandras, and D. L. Pepyne, "A backward algorithm for computing optimal controls for single-stage hybrid manufacturing systems," Int. J. Prod. Res., vol. 39-2, pp. 369-393, 2001.

[7] C. G. Cassandras and K. Gokbayrak, "Optimal control for discrete event and hybrid systems," Modeling, Control and Optimization of Complex Systems, pp. 285-304, 2002.

[8] Y. C. Cho, C. G. Cassandras, and D. L. Pepyne, "Forward decomposition algorithms for optimal control of a class of hybrid systems," Intl. J. of Robust and Nonlinear Control, vol. 11, pp. 497-513, 2001.

[9] C. G. Cassandras, D. L. Pepyne, and Y. Wardi, "Optimal control of a class of hybrid systems," IEEE Trans. on Automatic Control, vol. AC46,3 , pp. 398-415, 2001.

[10] K. Gokbayrak and C. G. Cassandras, "Stochastic optimal control of a hybrid manufacturing system model," Proc. of 38 th IEEE Conf. Decision and Control, pp. 919-924, 1999.

[11] D. P. Bertsekas, Dynamic Programming and Optimal Control. Belmont, Massachusetts: Athena Scientific, 1995.

[12] O. Selvi and K. Gokbayrak, "Stochastic optimal control of a two-stage hybrid manufacturing system," Tech. Rep. 2005-01, Bilkent University, Industrial Engineering Department, 2005.

[13] M. Mitchell, An Introduction to Genetic Algorithms. The MIT Press, 1998. 